\title{
Formation of Rydberg Atoms in an Expanding Ultracold Neutral Plasma
}

Scott D. Bergeson

scott.bergeson@byu.edu

T. C. Killian

M. J. Lim

S. Kulin

R. Dumke

See next page for additional authors

Follow this and additional works at: https://scholarsarchive.byu.edu/facpub

Part of the Astrophysics and Astronomy Commons, and the Physics Commons

\section{Original Publication Citation}

T. C. Killian, M. J. Lim, S. Kulin, R. Dumke, S. D. Bergeson, and S.L. Rolston, "Formation of Rydberg atoms in an expanding ultracold neutral plasma," Physical Review Letters, 86, 3759-3762 (21). The original publication of this article can be found at http://link.aps.org/doi/ 1.113/PhysRevLett.86.3759

\section{BYU ScholarsArchive Citation}

Bergeson, Scott D.; Killian, T. C.; Lim, M. J.; Kulin, S.; Dumke, R.; and Rolston, S. L., "Formation of Rydberg Atoms in an Expanding Ultracold Neutral Plasma" (2001). Faculty Publications. 574.

https://scholarsarchive.byu.edu/facpub/574

This Peer-Reviewed Article is brought to you for free and open access by BYU ScholarsArchive. It has been accepted for inclusion in Faculty Publications by an authorized administrator of BYU ScholarsArchive. For more information, please contact ellen_amatangelo@byu.edu. 


\section{Authors}

Scott D. Bergeson, T. C. Killian, M. J. Lim, S. Kulin, R. Dumke, and S. L. Rolston 


\title{
Formation of Rydberg Atoms in an Expanding Ultracold Neutral Plasma
}

\author{
T. C. Killian, M. J. Lim, S. Kulin, R. Dumke, S. D. Bergeson,* and S. L. Rolston \\ National Institute of Standards and Technology, Gaithersburg, Maryland 20899-8424
}

(Received 30 November 2000)

\begin{abstract}
We study the formation of Rydberg atoms in expanding plasmas at temperatures of $1-1000 \mathrm{~K}$ and densities from $10^{5}-10^{10} \mathrm{~cm}^{-3}$. Up to $20 \%$ of the initially free charges recombine in about $100 \mu \mathrm{s}$, and the binding energy of the Rydberg atoms approximately equals the increase in the kinetic energy of the remaining free electrons. Three-body recombination is expected to dominate in this regime, yet most of our results are inconsistent with this mechanism.
\end{abstract}

DOI: 10.1103/PhysRevLett.86.3759

Recombination of electrons and ions to form neutral atoms is a fundamental process in plasma physics. The flow of electrons between bound and unbound states must be understood in order to optimize lighting sources and commercial processing, or to model astrophysical chemistry or fusion reactors. At typical plasma temperatures of $1000 \mathrm{~K}$ or higher, radiative recombination (RR), dielectronic recombination (DR), and three-body recombination (TBR) are the dominant processes, and theory and experiment agree well [1,2]. RR [3], in which an electron is captured and a photon is released, populates tightly bound levels and dominates at densities below $10^{14} \mathrm{~cm}^{-3}$. DR [1] can be seen as a variation of this process, in which an intermediate excited electronic state is formed before radiative decay occurs. TBR [4], in which an electron and an ion recombine and a second electron serves to conserve energy and momentum, becomes important at higher densities and populates high lying Rydberg atomic states.

The situation changes drastically at temperatures of order one Kelvin, which can be realized in an ultracold neutral plasma produced by photoionization of laser-cooled atoms [5]. The total three-body rate is predicted to vary with temperature as $T^{-9 / 2}$, implying fast recombination that dominates over RR and DR even at moderate densities of $10^{10} \mathrm{~cm}^{-3}$ or lower. This divergent temperature dependence has inspired investigations into whether TBR theory must be modified in this new regime [6-8]. In ion storage rings, collisions between ions and electrons in electron coolers can be in the 10-100 K energy range, and anomalously large recombination rates have been observed for highly charged ions [9]. Some authors have suggested an enhanced contribution from TBR to explain a portion of the excess [10]. In addition to the fundamental interest in TBR at low temperatures, the process has a possible application in the formation of cold antihydrogen through positron-antiproton recombination [11]. We note that the inverse process, the spontaneous evolution of a cold gas of Rydberg atoms into a plasma, has recently been reported [12].

We have observed recombination into Rydberg atoms in an ultracold neutral plasma. At temperatures ranging from $1-1000 \mathrm{~K}$, and densities from $10^{5}-10^{10} \mathrm{~cm}^{-3}$, up to $20 \%$
PACS numbers: 34.80.Lx, 32.80.Pj, 52.20.-j, 52.55.Dy

of the initially free charges recombine on a time scale of $100 \mu \mathrm{s}$. The Rydberg atom distributions as a function of principal quantum number and time are determined for a wide range of initial conditions, allowing us to compare the recombination rate with existing theory. This study was motivated by our recent observation [13] of anomalous heating of electrons under cold and dense plasma conditions.

The creation of an ultracold plasma has been described in [5]. Approximately $5 \times 10^{6}$ metastable xenon atoms are laser cooled to $10 \mu \mathrm{K}$. The peak density is about $5 \times 10^{10} \mathrm{~cm}^{-3}$, and the spatial distribution of the cloud is Gaussian with an rms radius $\sigma \approx 180 \mu \mathrm{m}$. To produce the plasma, up to $25 \%$ of the atoms are photoionized in a $10 \mathrm{~ns}$ two-photon excitation. Because of the small electronion mass ratio, the resulting electrons have an initial kinetic energy $\left(E_{e}\right)$ approximately equal to the difference between the photon energy and the ionization potential. In this study we vary $E_{e} / k_{B}$ between 1 and $1000 \mathrm{~K}$. The initial kinetic energy of the ions varies between $10 \mu \mathrm{K}$ and $4 \mathrm{mK}$.

Figure 1 shows the electron signal from an ultracold neutral plasma created by photoionization at $t=0$. A $5 \mathrm{mV} / \mathrm{cm} \mathrm{dc}$ field, applied using grids located above and below the laser-atom interaction region, directs electrons to a multichannel plate for detection. Some electrons leave the sample and arrive at the detector within a few $\mu$ s, producing the first peak in the signal. The ions are essentially immobile on this time scale, and the resulting excess positive charge in the plasma creates a Coulomb potential well that traps the remaining electrons [5]. In the work reported here, typically $>80 \%$ of the electrons are trapped, and the plasma density profile is initially similar to that of the laser-cooled atom cloud. As the plasma expands [13], the depth of the Coulomb well decreases, allowing the remaining free electrons to leave the trap. This produces the broad feature in Fig. 1 centered at $t \approx 50 \mu \mathrm{s}$.

After the free electrons have escaped, the electric field is increased to $120 \mathrm{~V} / \mathrm{cm}$ in $50-100 \mu \mathrm{s}$. This field can ionize Rydberg atoms bound by as much as $70 \mathrm{~K}$, corresponding to a principal quantum number of about $n=47$. From the number of electrons reaching the detector we 


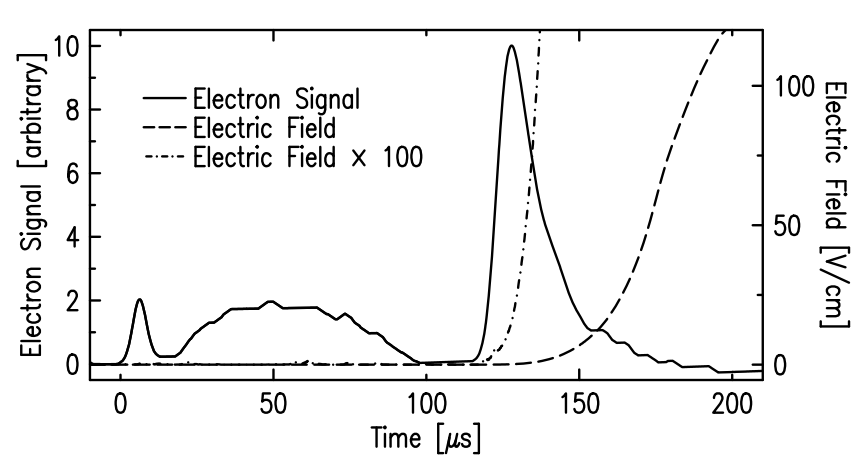

FIG. 1. Electron signal from a plasma created by photoionizing $10^{5}$ atoms at $t=0$, with $E_{e} / k_{B}=206 \mathrm{~K}$. The first and second features represent free electrons escaping from the plasma. The third feature arises from ionization of Rydberg atoms. A $5 \mathrm{mV} / \mathrm{cm}$ field is present before the large field ramp commences at about $120 \mu \mathrm{s}$, and the collection and detection efficiency for the first and second features is approximately $10 \%$ of the efficiency for electrons from Rydberg atoms.

infer the number of Rydberg atoms formed, and from the field at which the atoms ionize we construct the distribution of Rydberg atoms as a function of $n$.

We find that the Rydberg atoms survive for hundreds of microseconds with no significant change in their distribution. This implies that most of the atoms are in high angular momentum states (high $l$ ) that have long radiative lifetimes [14,15].

During field ionization, as the electric field increases, atomic levels shift due to the Stark effect, and there are many level crossings before atoms ionize. At the high $n$ and $l$ of this work, interactions between levels are small, and even for the slow field ramp used, atoms traverse the crossings diabatically, without changing state. For a given $n$, the distribution of field values at which different sublevels ionize begins at $F_{\text {diab }}=F_{0} /\left(9 n^{4}\right)$, where $F_{0}=5.14 \times 10^{9} \mathrm{~V} / \mathrm{cm}[14]$, and has a tail that extends up to 2-3 times higher [16]. Simulations based on the decay rates of hydrogenic Stark states [17] indicate that for equal population of all sublevels, the average ionization field exceeds $F_{\text {diab }}$ by about $50 \%$. We will use this average result when determining Rydberg population distributions from the ionization signals.

Figure 2 shows typical data. As the number of atoms photoionized $\left(N_{i}\right)$ increases, or $E_{e}$ decreases, a greater fraction of charges recombine and the Rydberg distribution shifts toward more deeply bound levels. The integral of each curve yields the total number of Rydberg atoms formed, which can reach a fraction of 0.2(1) of the initial number of ionized atoms [18]. The expected rates for RR or DR are many orders of magnitude too low to account for the observed Rydberg atom formation. We turn to TBR, which is expected to dominate at ultracold temperatures.

At electron temperature $T_{e}$, the TBR rate per volume $[6,19]$ into a given $n$ can be written as

$$
\dot{\rho}_{n}=C \rho_{e}^{2} \rho_{\mathrm{ion}} T_{e}^{-1} n^{4}\left(1-e^{-x_{n}}\right)\left(\frac{g_{n}}{g_{e} g_{\mathrm{ion}}}\right),
$$

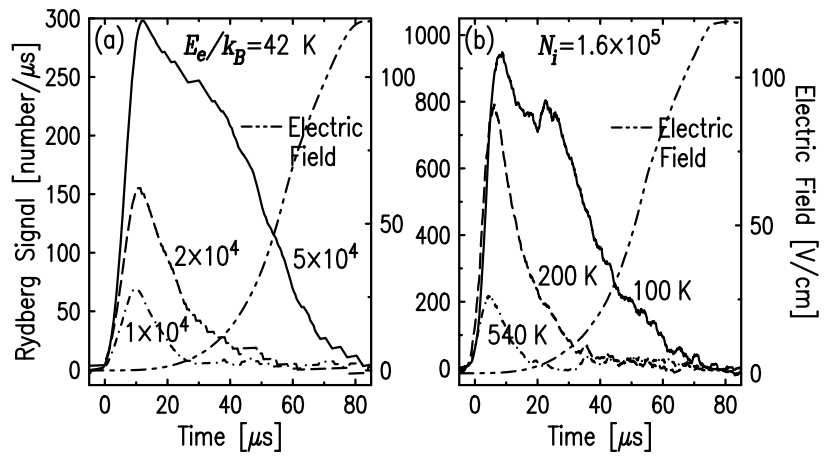

FIG. 2. Rydberg ionization signals for various plasma conditions approximately $100 \mu \mathrm{s}$ after photoionization. The time origin is the start of the electric field ramp. (a) Constant $E_{e} / k_{B}=42 \mathrm{~K} . N_{i}$ is indicated near each curve. (b) Constant $N_{i}=1.6 \times 10^{5} . E_{e} / k_{B}$ is indicated near each curve.

where $C=4.6 \times 10^{-38} \mathrm{~m}^{6} \mathrm{~K} \mathrm{~s}^{-1}$. All quantities in this equation are in SI units (including density in $\mathrm{m}^{-3}$ ) and $x_{n}=E_{n} / k_{B} T_{e}$, where $E_{n}=E_{1} / n^{2}$ is the binding energy of level $n$. $\left(E_{1}=2.18 \times 10^{-18} \mathrm{~J}\right.$ is the ionization potential of hydrogen.) The degeneracies are $g_{e}=2$ for electrons, and $g_{\text {ion }}=4$ for ions (total angular momentum of $3 / 2$ ). An ion also forms the core of each Rydberg atom, for which $g_{n}=8 n^{2}$ [20].

Models of population distributions in equilibrium plasmas take into account TBR through Eq. (1), collisional ionization, and collisional and radiative population redistribution. They predict a density-independent maximum in the Rydberg atom distribution at levels bound by a few $k_{B} T$ [2]. This contradicts the trend observed in this experiment towards more deeply bound levels as $N_{i}$ increases or $E_{e}$ decreases (Fig. 2).

Data taken after all recombination has ceased, such as in Fig. 2, is difficult to quantitatively analyze because the electron temperature may change significantly during the plasma expansion. By measuring Rydberg atom distributions at various times after photoionization [21], however, we can isolate and analyze particular features of the recombination process. Figure 3 shows such data for a plasma with high $N_{i}$ and low $E_{e}$ [22]. By $12 \mu \mathrm{s}$, there are large numbers of Rydberg atoms in low $n$ states $(n<$ 60 ). The corresponding recombination rates per level of $\sim 5 \times 10^{7} \mathrm{~s}^{-1}$ in this time interval agree with Eq. (1) evaluated for the initial plasma conditions. For higher $n$, Eq. (1), which scales as $n^{6}$, predicts many more Rydberg atoms than observed. This suggests that the electron thermal energy is comparable to or exceeds the binding energies, and collisional ionization is destroying Rydberg atoms as fast as they are created. The expected Saha equilibrium distribution [19] agrees well with high $n$ data.

From 12-22 $\mu \mathrm{s}$, there is a net loss of Rydberg atoms. This is consistent with decreasing density, which pushes equilibrium towards fewer neutral atoms provided the temperature does not drop significantly. We know from [13] that a plasma with these initial conditions expands with a velocity of about $60 \mathrm{~m} / \mathrm{s}$, and the density will decrease as 


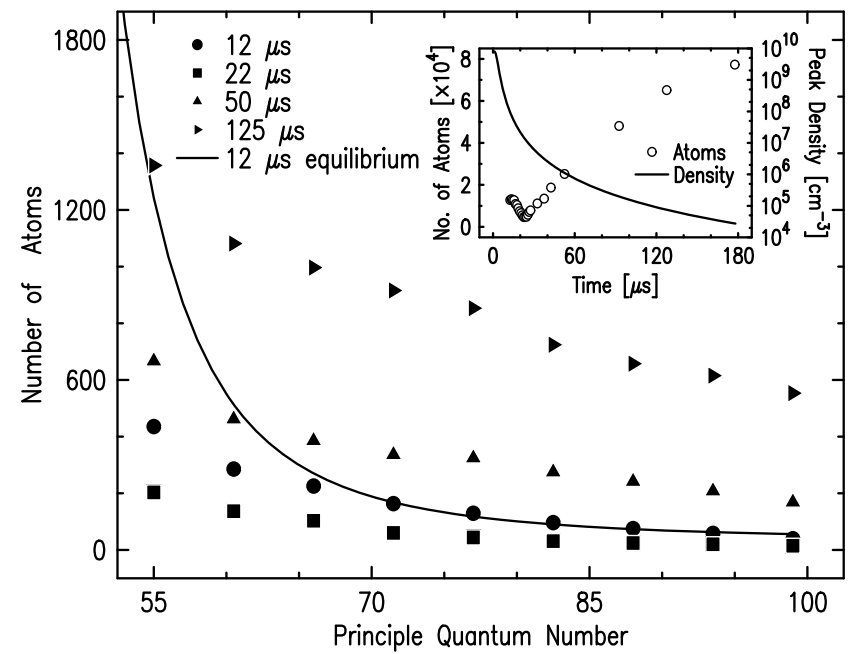

FIG. 3. Rydberg atom distributions at various times after photoionization for $E_{e} / k_{B}=9 \mathrm{~K}$ and $N_{i}=7 \times 10^{5}$. The $12 \mu \mathrm{s}$ Saha equilibrium distribution shown is for $T_{e}=8.5 \mathrm{~K}$ and a Gaussian plasma density distribution with rms size $\sigma=600 \mu \mathrm{m}$. These are reasonable conditions for the $12 \mu \mathrm{s}$ data. The Rydberg atom distributions at $t \geq 50 \mu \mathrm{s}$ do not match the equilibrium distribution for any reasonable parameters. The inset shows the total number of Rydberg atoms observed as a function of time and the peak density in the plasma for an expansion velocity of $v_{0}=60 \mathrm{~m} / \mathrm{s}$ [13]. There is a $10 \%$ uncertainty in the assigned quantum numbers due to the diabatic ionization, and there is a factor of 2 scale uncertainty in the number of Rydberg atoms [18].

shown in the inset in Fig. 3. In TBR, the extra electron involved, which remains free in the plasma, gains the binding energy as kinetic energy. The binding energy of the Rydberg atoms is significant compared to $E_{e}$ and could offset cooling due to adiabatic expansion, or even cause initial heating of the electrons.

Surprisingly, from $22-125 \mu$ s the Rydberg atom population increases again as the plasma density decreases below $\rho \approx 10^{6} \mathrm{~cm}^{-3}$. The observed recombination rate for $n=55$ is $\sim 10^{7} \mathrm{~s}^{-1}$ during this time. To account for this with a naive application of standard TBR theory embodied in Eq. (1), the temperature would need to drop below $1 \mathrm{mK}$. Such extreme cooling is possible. Neglecting heating due to Rydberg atom formation and using a polytropic exponent of $\gamma=5 / 3$ [23,24], adiabatic expansion of the plasma would decrease the electron temperature to about $30 \mathrm{mK}$ by $50 \mu \mathrm{s}$. The escape of electrons out of the trap formed by the ions, such as shown in Fig. 1 from 20-100 $\mu \mathrm{s}$, could further reduce the temperature of the electrons through evaporative cooling [25]. However, at such a low temperature the system would have an electron Coulomb coupling parameter [26] $\Gamma_{e} \approx 300$. It is unknown how a neutral plasma would behave in this regime; single component systems with such strong coupling behave as solids and minimize their potential energy by forming Wigner crystals [27]. In this regime standard TBR and classical plasma theory are invalid.

Perhaps recombination in expanding ultracold plasmas proceeds through a qualitatively different mechanism. Liq- uidlike spatial correlations between particles develop as the system cools to $\Gamma_{e} \approx 1$. Through a many-body process, spatially correlated ions and electrons could freeze out during plasma expansion, resulting in Rydberg atoms. A detailed model of the thermodynamics of the expanding plasma or an experimental probe of the temperature of the electrons is needed to explore these possibilities.

Plasmas with lower $N_{i}$ and higher $E_{e}$ than represented in Fig. 3 form fewer prompt $(t<20 \mu \mathrm{s})$ Rydberg atoms and exhibit little or no indication of equilibrium. Also, the distributions shift to higher $n$. An increasing recombination rate at $t>20 \mu \mathrm{s}$, however, is common to all initial experimental conditions.

An important piece of information that can be extracted from the data is the total binding energy released during the formation of Rydberg atoms. To conserve energy, this must be balanced by an increase in energy in some other part of the system. The hydrodynamic model developed in [13] shows that the pressure of the electron gas drives the plasma expansion, and the expansion velocity is a sensitive probe of the electron thermal energy at early times. For initial $\Gamma_{e} \lesssim 0.1$, the thermal pressure was observed to be characteristic of the energy available from the photoionization, $E_{e}$. At higher $\Gamma_{e}$ (higher density and lower temperature) the plasma expansions were faster than expected for a given $E_{e}$, indicating the existence of an additional source of electron thermal energy. By plotting the Rydberg binding energy on the same graph as the excess expansion energy (Fig. 4), we see that within the uncertainty [18], the two energies are equal for $\Gamma_{e} \lesssim 0.5$. Data from several different detector configurations and calibrations are shown.

The Rydberg data in Fig. 4 does not extend as high in $\Gamma_{e}$ as the expansion data does because as either $E_{e}$ decreases or $N_{i}$ increases, the Rydberg distributions shift towards lower $n$, and eventually significant population is in more deeply bound levels than the field ramp can ionize. The observed Rydberg distributions then yield an underestimate

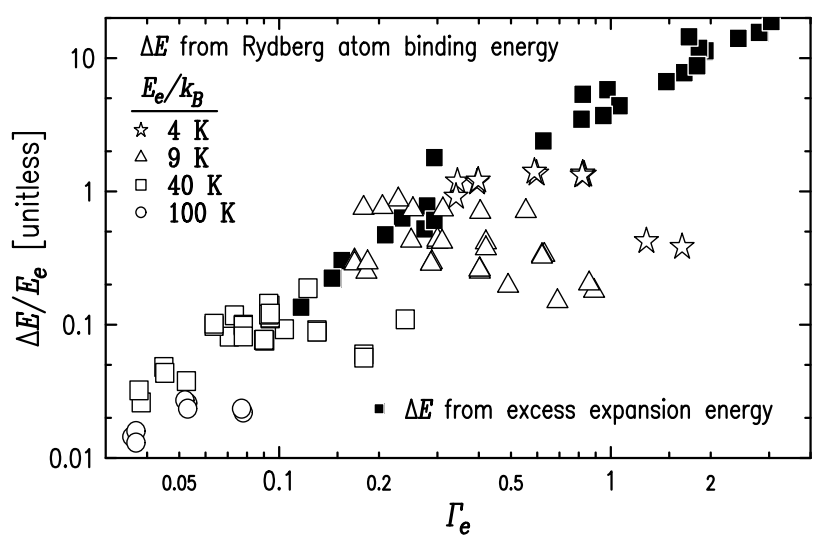

FIG. 4. The excess energy in the plasma expansion (from [13]) approximately equals the binding energy of the observed Rydberg atoms. $\Delta E$ is the total energy observed divided by the number of ions initially formed. $\Gamma_{e}$ is the initial coupling parameter, calculated by setting $3 k_{B} T_{e} / 2=E_{e}$. 
of the total binding energy. This limits the measurement to $\Gamma_{e} \lesssim 0.5$. The problem is also evident at lower $\Gamma_{e}$ for high $N_{i}$, as seen in the rollover of Rydberg data for a given $E_{e}$.

The equivalence of the excess expansion energy and the Rydberg binding energy suggests that the recombination is associated with the heating of the remaining free electrons. TBR would produce an energy balance similar to the one observed because the extra electron takes the binding energy as kinetic energy. It is important to note, however, that the heating occurs before most of the Rydberg atoms are formed. In [13] the electron heating was evident in the plasma expansions by $20-30 \mu \mathrm{s}$. In the present experiment the Rydberg atom distribution is still changing significantly at this time (see Fig. 3), and the total binding energy is typically only $20 \%$ of its final value. The Rydberg distributions analyzed in Fig. 4 were taken after all recombination was completed, approximately $100 \mu \mathrm{s}$ after photoionization. The time delay between the appearance of excess energy in the expansion and the formation of Rydberg atoms suggests that the recombination mechanism is more complicated than just TBR.

We have observed the formation of large numbers of Rydberg atoms in ultracold neutral plasmas. High recombination rates at low density can be explained with existing theory only if the electron temperature drops below $1 \mathrm{mK}$ during the expansion. Even if such extreme cooling does occur, other data appear to be at odds with three-body recombination - the increase in the average binding energy of the Rydberg atoms with decreasing $E_{e}$ and increasing $N_{i}$, and the time delay between the increase in the expansion velocity and the formation of Rydberg atoms. A detailed model is required to quantitatively explain all the observations, and we hope this study will motivate more theoretical work on recombination and plasma dynamics in this regime.

This work was funded by the Office of Naval Research.

*Present address: Department of Physics and Astronomy, Brigham Young University, Provo, UT 84602-4640.

[1] Recombination of Atomic Ions, edited by W. Graham, W. Fritsch, Y. Hahn, and J. A. Tanis, NATO ASI, Ser. B, Vol. 296 (Plenum, New York, 1992).

[2] J. Stevefelt, J. Boulmer, and J-F. Delpech, Phys. Rev. A 12, 1246 (1975).

[3] M. J. Seaton, Mon. Not. R. Astron. Soc. 119, 81 (1959).

[4] P. Mansbach and J. Keck, Phys. Rev. 181, 275 (1969).

[5] T. C. Killian et al., Phys. Rev. Lett. 83, 4776 (1999).

[6] Y. Hahn, Phys. Lett. A 231, 82 (1997).

[7] Y. Hahn, Phys. Lett. A 264, 465 (2000).
[8] S. A. Maiorov, A. N. Tkachev, and S. I. Yakovlenko, Phys. Scr. 51, 498 (1995).

[9] H. Gao et al., Phys. Rev. Lett. 75, 4381 (1995); G. Gwinner et al., Phys. Rev. Lett. 84, 4822 (2000).

[10] M. Pajek and R. Schuch, Phys. Scr. T80, 307 (1999).

[11] G. Gabrielse, S. L. Rolston, L. Haarsma, and W. Kells, Phys. Lett. A 129, 38 (1988); G. Gabrielse et al., Phys. Lett. B 455, 311 (1999).

[12] M. P. Robinson et al., Phys. Rev. Lett. 85, 4466 (2000).

[13] S. Kulin, T. C. Killian, S. D. Bergeson, and S. L. Rolston, Phys. Rev. Lett. 85, 318 (2000).

[14] T. Gallagher, Rydberg Atoms (Cambridge University Press, Cambridge, 1994).

[15] For high $l$ states, the lifetime scales as $n^{2}$ and is limited by blackbody radiation [14]. Although the lifetime is already $100 \mu \mathrm{s}$ for $n=50$, transitions are predominantly to near lying levels, so blackbody radiation will not significantly affect our results.

[16] F. G. Kellert et al., Phys. Rev. A 23, 1127 (1981); J. H. M. Neijzen and A. Dönszelmann, J. Phys. B 15, L87 (1982).

[17] R. J. Damburg and V. V. Kolosov, J. Phys. B 12, 2637 (1979).

[18] A factor of 2 uncertainty in the number of Rydberg atoms formed arises from detector calibration.

[19] D. R. Bates, A. E. Kingston, and R. W. P. McWhirter, Proc. R. Soc. London A 267, 297 (1962).

[20] The total recombination rate into all levels is often found by integrating Eq. (1) up to $n_{T}$, the Thomson value set by ionization by thermal electrons. At $n_{T}$ the binding energy is on the order of the thermal energy, $E_{1} / n_{T}^{2} \approx k_{B} T_{e}$. This yields a total rate that varies as $T_{e}^{-9 / 2}$.

[21] After allowing recombination to proceed for the desired length of time, we apply a 5-10 $\mu$ s long, $6 \mathrm{~V} / \mathrm{cm}$ electric field pulse to sweep away the plasma. This terminates the recombination process because all of the free charges are extracted. By removing the plasma before the large field ramp is applied, we also avoid saturation of the detector. This procedure ionizes Rydberg atoms with $n>100$.

[22] Each data point is obtained by scaling the electron signal and averaging over a range of electric field strength corresponding to a bin of five principle quantum numbers.

[23] L. Spitzer, Jr., Physics of Fully Ionized Gases (John Wiley \& Sons, Inc., New York, 1962), Chap. 5.

[24] Ch. Sack and H. Schamel, Phys. Rep. 156, 311 (1987); G. Manfredi, S. Mola, and M. R. Feix, Phys. Fluids B 5, 388 (1993); H.-B. Valentini and E. Glauche, J. Phys. D 28, 2279 (1995).

[25] W. Ketterle and N. J. Van Druten, Adv. At. Mol. Opt. Phys. 37, 181 (1996).

[26] S. Ichimaru, Rev. Mod. Phys. 54, 1017 (1982). The Coulomb coupling parameter is the ratio of the Coulomb and thermal energies for electrons, $\Gamma_{e}=$ $\left(e^{2} / 4 \pi \varepsilon_{0} a\right) / k_{B} T_{e}$. Here $a=(4 \pi \rho / 3)^{-1 / 3}$ is the WignerSeitz radius and $\rho$ is the peak density.

[27] For a review see Invited and Tutorial Papers from the 40th Annual Meeting of the Division of Plasma Physics of the APS [Special Issue of Phys. Plasmas 6 (1999)]. 